\title{
Construction of Ways to Improve College English Teachers' Informationized Teaching Ability Under the Background of "Internet+"
}

\author{
Feng Xin
}

School of Humanities, Heyuan Polytechnic, Heyuan, Guangdong, China

13268344@qq.com

\begin{abstract}
This paper adopts the method of literature and observation. This paper studies the ways to improve college English teachers' informationized teaching ability under the background of "Internet + " from three dimensions, i.e. the basic connotation and features of "Internet+"; interpretation of English teachers' information-based teaching ability; the analysis of the current situation of college English teachers' informationized teaching ability. The results show that: for the weak current information teaching consciousness and poor ability of information-based teaching, it is necessary to improve the quality of school information teaching infrastructure and enrich the information teaching environment. Improving English teachers' ability to use multimedia information technology in teaching; getting various ways to enhance English teachers' awareness of information-based teaching; promoting teachers' self-improvement; perfecting the construction of college English teachers' informatization teaching ability under the background of "Internet + " from four aspects. It provides reference suggestions for college English teachers to adapt themselves to the information age, improve their information teaching ability, and improve the effectiveness and teaching wisdom of English classes.
\end{abstract}

Keywords: "Internet+", college English teacher, information-based teaching, the path

\section{“互联网+”背景下高校英语教师信息化教学能力提升路 径构建}

冯 金金

河源职业技术学院人文学院, 河源, 广东, 中国

13268344@qq.com

摘要: 本文运用文献资料法、观察法。从 “互联网+” 基本内涵和基本特征; 英语教师信息化教学能力 解读; 高校英语教师信息化教学能力现状分析, 三个维度对 “互联网+” 背景下高校英语教师信息化教 学能力提升路径展开研究。结果表明：针对目前信息化教学意识不强; 信息化教学能力薄弱; 信息化 教学环境滞后等问题, 需要从提高学校信息化教学基础设施质量, 丰富信息化教学环境; 提升英语教 师使用多媒体信息化技术教学的能力; 多种方式增强英语教师信息化教学意识; 教师自我提升; 四个 方面完善 “互联网+” 背景下高校英语教师信息化教学能力提升路径的构建。为高校英语教师主动顺应 信息化时代，提高信息化教学能力，进而提高英语课堂有效性和教学智慧提供参考性建议。

关键词: “互联网+”，高校英语教师，信息化教学，路径

\section{1. 前言}

随着 “互联网+”时代的到来，新一轮的信息技术革 新也被开启。“互联网+” 背景下的教育环境新生态也在
不断地被创生。社会整体关注的焦点也逐渐向 “互联 网+”教育业聚焦。如何提升高校英语教师信息化的教 学能力, 也就自然成为教育理论界普遍关注的热点话 题。信息素养是 21 世纪重要的能力和终身学习能力的 重要前提。现代教育信息技术是教学能力的基本构成 部分，同时也是现代教师教育的核心课程之一，而当 
前教育信息化改革能否成功, 也主要在于教师信息素养 的提升。但是, 由于信息化教学和互联网信息技术是相 互依存, 彼此制约的双向关系, 任何一方的存在都是以 另一方的存在为基本前提的。本文通过构建高校英语教 师信息化教学水平提升的路径, 希望能够为科学合理的 提高英语教学质量和教学工作效率提供建议。

\section{2. “互联网 + ”阐释}

\section{1. “互联网 + ” 基本内涵}

“互联网+”是两两或者两多契合共融的升级版，把 目前信息媒体化前进的发展核心定格为互联网, 将此核 心提取出来，与工业、商业、金融业等服务业融合共生。 这其中的关键所在的源头就是创新, 只有抓住创新这个 源头, 才能让这个 “+” 赋有真正有意义和价值。所以说, “互联网+” 是创新 2.0 下对互联网发展新形态、新业态 的重新诠释, 是知识社会创新 2.0 背景下的新的经济形 态演进阶段。一般来讲, “互联网+”可以理解为 “互联 网+各个传统行业”, “但这并不是简单的两者相加, 而 是利用信息通信技术以及互联网平台, 让互联网与传统 行业进行深度融合。 ${ }^{[1]}$ ” 不断创生新的内需动力, 带动社 会飞速发展。

\section{2. “互联网 + ” 基本特征}

（1）是跨界融合: “+”就是跨越，就是革新，就 是解放, 就是重组契合。创新的基础要想更为坚实, 就 必须敢于跨界, 敢于融合。重组契合了, 集体智囊才会 突现, 从理想到实践的路径才会更通畅。(2) 是创新驱 动: 中国的源驱动型增长方式一直以来比较粗放, 不能 长久持续, 必须转变到创新驱动的进一步发展途径上来。 这也恰恰是互联网的特质, 用所谓的互联网思维来自我 蜕变和自我革新, 也更能激发创新的源泉。（3）是重组 重构: 技术革命、信息化、互联网化已打破了原有的结 构秩序。话语权阶段性地在发生变化。互联网+社会治理 既虚拟的网络化治理是对以往传统治理的巨大挑战。(4) 是尊重人性: 当前社会各个领域对于人品人性的要求已 经达到最高规格, 人品人性有问题, 绝对一票否决。良 好的人性是社会有秩序前进的最根本基石。“互联网的 力量之强大最根本地也来源于对人性的最大限度的尊 重、对人体验的敬畏、对人的创造性发挥的重视。 ${ }^{[1]}$ ”(5) 是开放生态: 生态是互联网特别重要的表征, 而生态的 本身就是开放的。我们崇尚推动 “互联网+” , 就是为了 解掉过去制约创新的阻碍因素, 把孤岛式创新点续传起 来。（6）是连接所有: 有层次的连接, 有差异连接, 但 是连接一切的终极目标是 “互联网+” 的背景下实现所有 技术的无缝衔接。

\section{3. 英语教师信息化教学能力解读}

随着 “互联网+” 时代的到来, “现代社会是属于 信息技术的社会, 各行各业都或多或少的渗透着信息 技术, 教育领域也不例外。 ${ }^{[2]}$ ”作为综合性的大系统的 学校教育教学系统, 信息技术在其中发挥着巨大的作 用。为了更好的发挥作用, 就需要每个教师要掌握必 要的信息技术。学校整体教学实力以及教学工作效率、 教学效果进步的前提是英语教师信息化教学能力的进 一步提升和完善，就此基础上， “钟志贤认为信息化 技能是各类教师知道如何利用计算机和网络技术以获 取相关教育信息, 以便对信息环境中的学习过程和学 习资源做出设计、应用、评价、管理的一种新型综合 教学能力。 ${ }^{[3]}$ ” 根据联合国教科文组织相关信息化教 学能力的解释, 信息化教学能力就是在学校整体的改 革与发展中融合进去现代化的多媒体信息技术，敦促 学校教育的科技化。科学技术是第一生产力, 所以也 应该让新的信息技术在教育教学领域发挥应有的作 用。也就是要求学校和教师吸收融合传统教学方式和 新技术创新教学方式的一切优势去构建更加完善的学 习环境, 与此同时, 学校应该制定导向政策, 引领教 师要积极开发创新式的多种方法融合的教学方式。其 中也应该把教师信息化教学能力作为政策导向的中心 环节。教师专业能力水平和教师自身的综合素质也可 以通过信息化教学能力来充分体现。信息技术已经渗 透在社会生活的层层面面，学校教育也无不例外的受 到教育信息化的强烈冲击。当然, 有市场就有竞争, 教育学校也是适者生存, 学校要想在教育领域占据自 己的一片市场, 就必须提高教师信息化教学能力, 转 变教育思想。教育科学工作者对教师教学能力的阐释 各抒己见, 我们需要倾听不同的声音, 而不是单一的 某个学者或者专家的观点。融合不同领域的专家对教 师教学能力阐发的观点, 当局者迷旁观者清, 跳出教 育去分析梳理教育, 才能全面综合认识到位。随着信 息化时代的普及，提高英语教师信息化教学能力已经 是势在必行。温家宝总理曾指出 “百年大计, 教育为 本; 教育大计, 教师为本”。从政策上导向学校既要 抓教师的一般素养也要抓教师专业能力, 两手抓, 两 手都要硬。在当前信息技术不断涌动的大思潮下, 教 师的信息化教学能力俨然已经成为其中重中之重的培 养目标。在教育的各个领域加强信息技术的推广，使 数字化、网络化、智能化、多媒体化在教学活动中争 相斗艳。以前传统教学教育的教师可以不精通信息化 教学, 但是当前社会下, 要求每个教师必须掌握这一 能力素质, 这就需要高校英语教师树立信息化教育观 念, 发挥自己教育领导者的主观优势, 用科学现代化 信息技术武装自己，推动学校现代化教育进程。教师 的一切是为了学生, 又是为了学生的一切, 其所有的 教学活动都是为了提升学生的综合素质, 订单式的培 养满足社会主义现代化建设的复合型新型人才。 


\section{4. 高校英语教师信息化教学能力现状分析}

和以往相比, 虽然当前高校英语教师信息化教学能 力已经有很大的进步, 但是与社会整体信息化的要求相 比, 还存在着巨大的差距, 突出地表现为教师信息化教 学意识不强, 能力薄弱, 信息化教学环境滞后。

\section{1. 信息化教学意识不强}

结合教育实践显示，教师的一切教育行为受到自身 意识限制。当前, 很多高校英语教师对待信息化教学只 是应付了事, 并没有意识到其在教学中的关键作用, 从 内心上排斥新兴信息化教学, 从而导致教师自觉主动性 较差, 总是以一定的经验主义进行一切教学活动。

\section{2. 信息化教学能力薄弱}

高校英语教师信息化教学能力是由多方面因素构 成, 每个因素之间又相互联系、相互制约, 并在每个因 素的相互作用下形成一个完整的系统性工程。这项系统 性的工程包含技术性和综合性两种特质。而目前, 不少 英语教师的信息化教学能力的构成还只是其中简单的基 础因素, 还处于初级阶段。教师资源整合意识薄弱, 能 力亟待提高, 对网络教学资源应用方式过于简单。并且, 教师设计建构信息化的能力薄弱。如表 1 , 随机抽取广东 省一所高校的英语教师对教学工具软件的运用能力程度 的调查结果显示, 英语教师对搜索网页和编辑文档的运 用能力较强, 对图片以及音频、视频剪辑的能力次之, 对网站、二三维的制作能力最差。信息技术要发挥整体 作用, 就必须使各个构成因素有机结合, 在共同达到教 学目标的过程中统一协调, 从客观实际出发, 针对不同 的对象和不同的水平进行不同的教学。而不是掌握几种 简单的操作方法, 就开始广泛的应用于教学中, 使得技 术运用过于简单, 效果不明显。

\section{表 1 高校英语教师对各种教学工具软件的运用能力状} 况

\begin{tabular}{|c|c|c|c|c|c|c|c|c|}
\hline \multirow{4}{*}{$\begin{array}{l}\text { 软件 } \\
\text { 应用 }\end{array}$} & 搜 & 编 & 美 & 视 & 音 & 网 & 二 & 三 \\
\hline & 索 & 辑 & 化 & 频 & 频 & 站 & 维 & 维 \\
\hline & 网 & 文 & 图 & 剪 & 剪 & 制 & 制 & 制 \\
\hline & 页 & 档 & 片 & 辑 & 辑 & 作 & 作 & 作 \\
\hline 平均 & 4.22 & 3.95 & 3.63 & 3.52 & 3.26 & 2.36 & 1.93 & 1.74 \\
\hline 分值 & & & & & & & & \\
\hline $\begin{array}{l}\text { 能力 } \\
\text { 排序 }\end{array}$ & 1 & 2 & 3 & 4 & 5 & 6 & 7 & 8 \\
\hline
\end{tabular}

\section{3. 信息化教学环境滞后}

要想教师信息化教学能力得到全面提升, 不仅要提 高教师信息技术运用能力和优化教师本身的知识构成, 同时还要一定的硬件环境来支撑才能有较好的教学效
果。随着政策的导向, 各个高校开始注重信息化教学 硬件设施的投入, 使得硬件环境得到了大幅改善。但 是, 内在的动因呢? 也就是促使教师进行信息化教学 的内在机制还有待提高, 我们称之为信息化教学的软 环境。比如：信息化教学氛围较差, 缺乏主动进行信 息化教学相关科研活动的动力; 资源环境硬件的投入 很简单, 但是投入了硬件却没有投入相关的指导培训, 或者没有引进专业的信息化教师做表率, 很大程度上 导致资源配置的浪费, 不能合理利用。

\section{5. “互联网+”背景下高校英语教师信息化 教学能力提升路径构建}

虽然当前教育界普遍关注的焦点是以教师为主 导, 学生为主体, 但是在真正的教学实践中是否能够 做到将课堂 “还给学生”、“以学生为中心呢? 而教 师的信息化教学能力仍旧是学生学习效果的重要因 素, 所以构建新时代 “互联网+” 背景下高校英语教师 信息化教学能力的提升路径势在必行。

\section{1. 多种方式增强英语教师信息化教学意 识}

教师信息化教学能力提升的前提首先是强化信息 化意识, 要提高意识就需要 “结合本校校情展开教师 教学能力职前培训。 ${ }^{[4]}$ ”结合自身的实际情况展开教 师教学能力职前培训。“培训的主要目的是使教师掌 握现代化教学资源的多重应用。同时可以定期举办教 师信息化教学能力相关的学术讨论来深入探讨和普及 信息化教学。 ${ }^{[5]}$ ” 首先可以举办教学比赛, 让教师们 以赛代练。比赛后, 组织英语教师们对各自在比赛中 发挥状况进行总结分析, 为他们创造良好的信息化教 学氛围。其次, 可以选择信息化开展较好的班级作为 先锋班, 充分发挥先锋班级以及先锋教师的模范带头 作用, 拓宽高校教师学习的路径。同时, 采取引进来 走出去的策略, 既提供丰厚的条件吸引信息化教学能 力名师、专家到校工作, 也可以选派自己学校愿意从 事信息化教学能力的英语教师参加培训等交流活动, 创造学习平台。

\section{2. 提升英语教师使用多媒体信息化技术 教学的能力}

要提升英语教师信息化教学能力, 首先需要教师 具备扎实的基本知识，同时也要了解当前先进的信息 化发展趋势, 使教师自己的知识储备和信息化的发展 趋势相匹配。其次, 还需要提高高校英语教师自身检 索信息、加工整合信息的能力，只有掌握了这些技术 手段才能提高他们的工作效率。随着英语教学信息的 丰富化, 教师需要对所搜集的教学信息进行一定的加 
工改造, 根据不同对象的不同水平, 选择切合实际的网 络信息资源进行教学。同时, 在 “互联网+” 背景下 “英 语教师们一定要正确使用信息化教学手段, 通过将传统 教学与其相互结合的方式, 来提高信息化教学能力。 ${ }^{[6]}$ ”

\section{3. 提高学校信息化教学基础设施质量, 丰富 信息化教学环境}

“高校英语教师信息化教学能力的提升还在于信息 化教学基础设施的完善。 ${ }^{[7]}$ ”比如教学资源库、教学案例 库、教务平台、观摩平台、多媒体教室、同步语音教室、 微格实验室等为支撑的硬件资源环境。能够为教师信息 化教学能力的提升构造良好的氛围。还可以采取奖励政 策, 激励学生和教师进行信息化教学。与此同时, “还 要对教师使用信息化硬件设施的能力进行培训, 让教师 能够熟练操作信息化教学设备。 ${ }^{[8]}$ ” 进而推动高校英语教 师的信息化教学能力的稳步提升。

\section{4. 教师自我提升}

信息化教学能力的提高需要英语教师做到以下几个 方面: 努力推动传统教学观念的革新, 关注对学生学习 活动的指导、计划、组织、协调, 不仅使学生知其然, 还要使学生知其所以然。结合每节课的具体教学目标和 学生的生理、心理特征等进一步制订 “互联网+” 背景下 信息化英语教学计划; “在信息化教学资源的实践运用 中, 切实做到目标定位、内容相符、对象适应。[9]” ; 提 高教师自身信息化教学的素养, 能够熟练地操作与英语 教学领域密切相关的网络信息化技术与设备; 帮助学生 充分运用网络媒体、期刊杂志、英语角等渠道来进行自 主学习, 培养学生的创造性思维和自我探索能力。

\section{6. 结论}

综上所述, “教师信息化教学能力成为英语教学革 新发展的重要制约因素。[10]”随着各行各业信息化技术 的不断渗入, 高校英语教师要努力提升自己的信息化教 学能力, 加强教学的信息化意识, 加强高校信息化教学 的基础设施硬件建设。总之, 高校英语教师要学会积极 主动地迎合教学信息化时代的切入，使自己的英语信息 化教学能力不断提升, 才能不被 “互联网+” 的新时代所 摒弃, 才能不断推动英语教学的革新。同时, 从事物发 展的内因寻求更好的行为解释, 进一步提升教师对信息 化教学的认识, 使之成为自我发展的核心内容, 并借助 不断的学习和培训等, 提高对信息技术和英语教学的加 工改造能力, 使得信息化教学成为自己的教学秘书, 让 英语教学在信息技术的深入融合中更上一层楼。

\section{REFERENCES}

[1] Xu, K. (2019) Research on the Management Model of Extra-curricular Sports Activities in Universities under the background of "Internet +". Nanjing: Nanjing Normal University.

[2] Cui, Y.L. (2014) Research on the Improvement of English Teachers' Information-based Teaching Ability. Chinese Journal of Education, 05:76-79.

[3] Zhong, Z.X., Wang, Y.M., Huang, Y., Shi, H.B. (2003) Investigation and Research on information literacy of primary and secondary school Teachers. Journal of Electronic Education, 01:65-69.

[4] He, X.L. (2007) Design and Research on the Training Program of pre-service Teachers' Basic Teaching Ability. Jinhua: Zhejiang Normal University.

[5] Yang, J.Y. (2010) Testing Students' Ability of Using Information Resources to Improve the Quality of the Teaching and Educating Aiming at Enabling Freshmen to Make Better Use of Library-Based on the Research Done at Suqian College.Library,08:52-53.

[6] Ji, F. (2017) Research on the Improvement of English Teachers' Informational Teaching Ability in Vocational Colleges. Journal of Liaoning Vocational College, 09:83-85.

[7] Xie, R.Q. (2016) The Fostering of the Ability to Interpret Images and the Teaching of Art Appreciation in High Schools. Journal of aesthetic education, 01: 101-104

[8] AlRufai, M.H. (1976) Ability Transfer and the Teaching of Reading. Elt journal, XXX(3): N/A.

[9] Mary, H., Neville, A.K. (2010) Ability to use a book: further studies of middle school children: Part I Ability to use a book: the effect of teaching. Literacy, 11(3):13-18.

[10] Ronfard, S., Corriveau K.H. (2016) Teaching and preschoolers' ability to infer knowledge from mistakes. Journal of Experimental Child Psychology, 150:87-98. 